\title{
Energy, Christiaan Huygens, and the Wonderful Cycloid-Theory versus Experiment
}

\author{
Yuval Ben-Abu ${ }^{1,2, *,+}$, Ira Wolfson ${ }^{3,+}$, Haim Eshach ${ }^{4}$ and Hezi Yizhaq ${ }^{5}$ \\ 1 Department of Physics and Project Unit, Sapir Academic College, Sderot 79165, Israel \\ 2 Hemdat Hadarom Academic College of Education, Netivot 80200, Israel \\ 3 Department of Physics, Ben-Gurion University of the Negev, Beer Sheva Campus 84990, Israel; \\ beastraban@gmail.com \\ 4 The Department of Science and Technology Education, Ben-Gurion University of Negev, \\ Beer Sheva Campus 84990, Israel; uvba.1973@gmail.com \\ 5 Department of Solar Energy and Environmental Physics, Jacob Blaustein Institute for Desert Research, \\ Ben-Gurion University of the Negev, Sede-Boker Campus 84990, Israel; youvalda@post.bgu.ac.il \\ * Correspondence: yuvalb@sapir.ac.il \\ + These authors contributed equally to this work.
}

Received: 4 February 2018; Accepted: 8 April 2018; Published: 16 April 2018

\begin{abstract}
The cycloid is one of the most intriguing objects in the classical physics world, at once solving the brachistochrone and isochronous curve problems. Historically, the cycloid shape has been employed to great success in many physical contexts. We discuss one such case, presenting the longitude problem as a pathway into an in-depth discussion of the analytical solution of a point mass motion along a cycloid. The classical solution is presented, and the modifications needed for a rolling ball along a cycloid rail are made. A comparison is then made between the two cases, and we show that the difference in most physical cases between the point mass and the rolling ball is at most $\sim 7 \%$. Next, an experiment is presented in which the isochronous nature of the cycloid path is tested, to different degrees of success. The results are discussed and several possible origins of the discrepancy between the theory and the experimental results are identified. We conclude with a discussion of skidding and slipless rolling.
\end{abstract}

Keywords: cycloid; brachistochrone; point mass; motion

\section{Introduction}

In 1662 the Royal British Academy announced a large monetary prize for building a precise naval clock that would enable solving the problem of finding longitude in the high seas. One of the problems that made navigating in the oceans difficult was finding longitude. While finding latitude through simple astronomical observations was relatively easy, finding longitude was a most difficult task. The British navy that ruled the oceans paid a high price for it. On 22 October 1707, three British navy ships making their way from Gibraltar to England crashed into the rocks off one of the islands, $32 \mathrm{~km}$ from England's southwest edge. In this accident, 2000 British sailors were killed [1]. This loss emphasized the need to solve the longitude problem. In 1714, the British Parliament announced the Longitude Act, which offered a prize of 20,000 pounds (a huge amount of money at the time) to anyone who could solve the longitude problem [1-3]. Already in the second half of the 17th century, it was clear that the solution to the problem lay in building a precise clock. Because one hour equals 15 degrees, it was understood that the measurement needed to be exact to the number of seconds in an arc. Measuring the difference between the two clocks-one that shows the time in a home port and one that shows the local time on a ship—enabled finding longitude in a precise way [1,2]. The said 
clock also needed to overcome the changes in weather and ship movements. For example, a regular pendulum clock was influenced by the changes in temperature that caused changes in the length of the string and therefore also caused changes in the pendulum's cycle times [4].

The Dutch scientist Christiaan Huygens (1629-1695) responded to the challenge and decided to upgrade Galileo's pendulum clock. His idea was to build an isochronous curve, meaning, a curved lane, upon which the motion time of the ball would not be dependent on the starting point. This pendulum would enable the building of clocks that were more precise than regular pendulum clocks, in which a mass moves along a pathway shaped as a circular arc. Huygens worked on this problem and on developing clocks for a period of almost 40 years, between 1656 and 1693 . He succeeded in demonstrating that the desired curve was a cycloid (Figure 1)—one of the most famous curves in mathematics that also solves the brachistochrone curve problem-finding the quickest pathway between two points. On 7 January 1657, he wrote, "These days I have discovered a method of building clocks, by means of which it will be possible to measure time so precisely that it will be possible to measure longitude even in the ocean" [3]. As proof that the cycloid is an isochronous pathway, he published a book entitled Horologium oscillatorium in 1673. Huygens proved this argument in an ingenious way (see Appendix A) based on basic mechanical reasoning, without using infinitesimal mathematics [2-7].

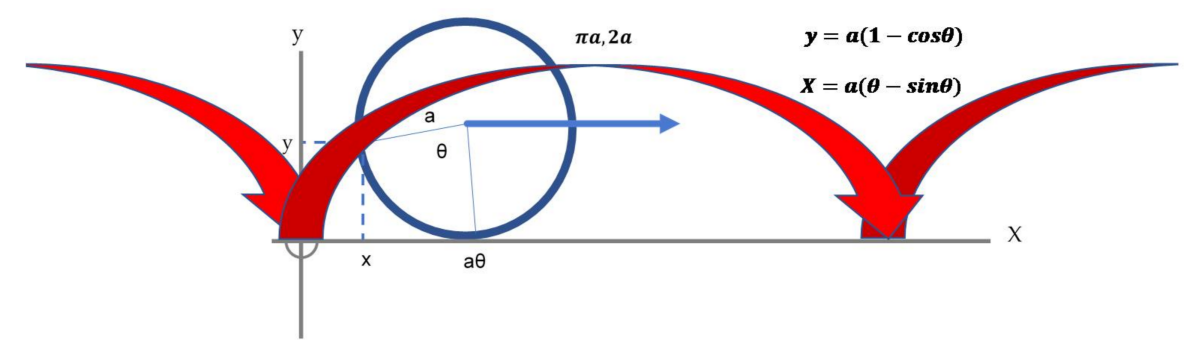

Figure 1. A cycloid is the curve that is created by the pathway of the point that is on the perimeter of the wheel without sliding. In this sketch, the parametric equations of a cycloid are presented, where a is the radius of the wheel and $\theta$ is the angle that is defined in the illustration. When the wheel completes a full circle, the angle changes from 0 to $2 \pi$.

In this manuscript, we explain Huygens' theory by comparing the experiment with the measurement of a ball's dependency on motion time from its starting height during its motion along different rails. This article is organized as follows: In the remainder of Section 1, we analytically discuss the cycloid and the usual approximations made, and we study their impact using computer simulations. Section 2 presents the methods and the experimental system, and Section 3 reviews the results. We conclude the paper in Section 4, and provide some appendices for the sake of thoroughness.

\subsection{The Cycloid: Coordinates, Equations of Motion, And Approximations}

In this section, we lay the theoretical groundwork for this article. We first derive the cycloid coordinates (Equation (4)). We then develop the Lagrangian and equations of motion for a point mass on a cycloid path, or, equivalently, a cycloid pendulum (Equation (6)); switch to canonical coordinates; and find the associated $\omega$ (Equation (11)). Finally, we add a rotational degree of freedom, which accounts for the slipless roll dynamics, and, in turn, for the energy stored in the rotation of the ball (Equations (14) and (15)). This is the framework necessary for understanding the isochronous movement and for making sense of the experiments described in the next sections.

\subsection{Cycloid Coordinates}

The cycloid form is derived by tracking a point on the circumference of a spinning wheel moving along a plane. Tracking a point on the circumference relative to the wheel's center, where the wheel is spinning clockwise, is given by 


$$
\left\{\begin{array}{c}
x=\operatorname{acos}(\theta) \\
y=-\operatorname{asin}(\theta)
\end{array}\right.
$$

Adding the motion of the wheel on the plane, we have the following connection between $x_{c m}$ and the rotation angle:

$$
x_{c m}=a \theta
$$

Thus, we are left with the following cycloid coordinates:

$$
\left\{\begin{array}{l}
x=a(\theta+\cos (\theta)) \\
y=a(1-\sin (\theta)) .
\end{array}\right.
$$

The introduction of a phase shifts these coordinates to a more canonical form:

$$
\left\{\begin{array}{l}
x=a(\theta+\sin (\theta)) \\
y=a(1-\cos (\theta))
\end{array}\right.
$$

which reach phase and $x_{0}$ calibration identical to the ones in Figure 1.

\subsection{Equations of Motion for a Cycloid}

Perhaps the best way to develop the equations of motion for the cycloid motion is through Lagrangian mechanics. This has the added value, as we shall show, of identifying generalized coordinates that simplify the problem greatly. We emphasize that in this following treatment we neglect the rotational degree of freedom. The kinetic term is simply given by

$$
\boldsymbol{K}=\frac{m v^{2}}{2}=\frac{m}{2}\left(\dot{x}^{2}+\dot{y}^{2}\right)=m a^{2} \dot{\theta}^{2}[1+\cos (\theta)]
$$

The potential energy $V$ is given by

$$
V=m g y=m g a[1-\cos (\theta)]
$$

Thus, the full Lagrangian is given by

$$
\mathcal{L}=m a^{2} \dot{\theta}^{2}[1+\cos (\theta)]-m g a[1-\cos (\theta)] .
$$

The above Lagrangian yields the following equation of motion, and angular momentum:

$$
\left\{\begin{array}{c}
\ddot{\theta}=-\frac{g}{a}\left[\frac{\sin (\theta)}{1+\cos (\theta)}\right] \\
P_{\theta}=L=m a^{2} \dot{\theta}[1+\cos (\theta)] .
\end{array}\right.
$$

Note that the angular velocity is not a conserved quantity. This is a good reason to find the conserved quantities in this system.

This Lagrangian can be written as

$$
\mathcal{L}=2 m a^{2} \dot{\theta}^{2} \cos ^{2} \frac{\theta}{2}-2 m g a \sin ^{2} \frac{\theta}{2}
$$

We now move to the following generalized coordinate $s$ :

$$
s=4 a \sin \frac{\theta}{2} \dot{s}=2 a \dot{\theta} \cos \frac{\theta}{2}
$$

This allows us to write the Lagrangian in the form

$$
\mathcal{L}=\frac{m \dot{s}^{2}}{2}-\frac{m g s^{2}}{8 a}
$$

which is nothing but a harmonic oscillator Lagrangian in s, with the associated angular velocity 


$$
\omega_{s}=\frac{1}{2} \sqrt{\frac{g}{a}} .
$$

Note that the angular velocity for $s$ is conserved and that $T=4 \pi=\sqrt{a / g}$.

\section{4. "Get the Ball Rolling" - Correcting for Angular Kinetic Energy}

Thus far, the equation of motion was simple and analytically solvable. Now, we add the rotation of the ball itself, with its radius $r$. The moment of inertia for a solid ball is given by (c.f. [5])

$$
I=\frac{2 m r^{2}}{5} \text {. }
$$

The connection between $\theta$, the cycloid angle, and the rotation of the ball around its central axis is given by

$$
r d \phi=a \sqrt{2+2 \cos (\theta)} d \theta \Rightarrow \omega=\frac{a \dot{\theta} \sqrt{2+2 \cos (\theta)}}{r} .
$$

So, the added term to the Lagrangian is given by

$$
K_{\omega}=\frac{I \omega^{2}}{2}=\frac{m r^{2} \omega^{2}}{5}=\frac{2 m a^{2}[1+\cos (\theta)] \dot{\theta}^{2}}{5} .
$$

The corrected Lagrangian is given by

$$
\mathcal{L}=\frac{7 m a^{2} \dot{\theta}^{2}[1+\cos (\theta)]}{5}-m g a[1-\cos (\theta)]
$$

The equation of motion is then given by

$$
\ddot{\theta}=\frac{\sin (\theta)}{14(1+\cos (\theta))}\left[7 \dot{\theta}^{2}-\frac{5 g}{a}\right]
$$

which, interestingly enough, does not seem to be dependent on the radius of the ball itself, nor on its mass.

\subsection{The Influence of Slipping While Rolling in Motion on Cycloid Pathways}

As mentioned above, the effect of rolling on the motion in a cycloid path is not dependent on the radius of the rolling ball. This can be seen in Equation (15), where the term for angular acceleration does not depend on $r$. This is a consequence of the connection between the pathway angle $\theta$ and the rolling angle of the ball $\phi$ as shown in Equation (13). An interesting question, then, is to what extent slipping while rolling might change the movement and the period.

In order to answer that, we find the equation of motion for rolling, while applying instead of Equation (13) a slightly different connection:

$$
\omega=(1-\alpha) \frac{a \dot{\theta} \sqrt{2+2 \cos (\theta)}}{r}
$$

where $\alpha$ is the relative part of the movement that is slipping; when there is no slipping, $\alpha=0$, and we should revert to a rolling without slipping. This is obviously a crude model which bundles the slipping into some fractional quantity, disregarding the position in which the slipping had occurred, etc.

The equations of motion are then given by

$$
\frac{d P_{\theta}}{d t}=-\sin (\theta)\left[\frac{5+2(1-\alpha)^{2}}{5} m a^{2} \dot{\theta}^{2}+m g a\right]
$$

where $\frac{d P_{\theta}}{d t}=\frac{2\left(5+2(1-\alpha)^{2}\right)}{5} m a^{2}\left[\left(1+\cos (\theta) \ddot{\theta}-\sin (\theta) \dot{\theta}^{2}\right)\right]$.

Now we add by hand the friction term to get

$$
\ddot{\theta}=\frac{\sin (\theta)}{1+\cos (\theta)}\left[\frac{\dot{\theta}^{2}}{2}-\left(\frac{\frac{5 g}{a}}{2\left(5+2(1-\alpha)^{2}\right)}\right)\left(1+\alpha \mu_{k} \cot (\alpha) \frac{\dot{\theta}}{|\dot{\theta}|}\right)\right] .
$$


One can easily see that when $\alpha=0$, we indeed revert back to the slipless roll case (Figure 2).

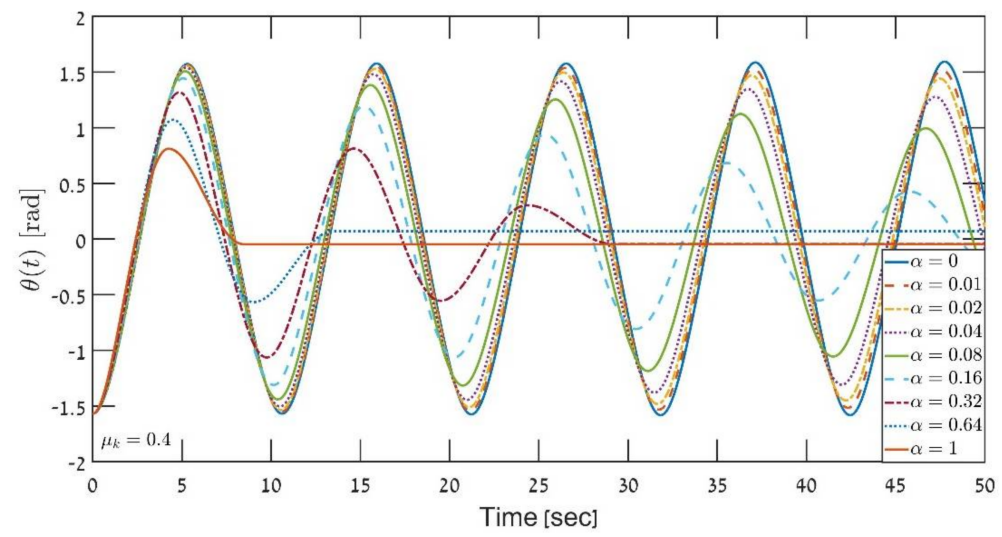

Figure 2. Rolling motion of a ball on a cycloid path, with slippage. $\alpha$ is the relative slipping factor, which represents how much of the movement is slipping and how much is slipless rolling. $\mu_{\mathrm{k}}$ was set to 0.4 , which is approximately the kinetic friction coefficient for steel on steel.

\section{Methods}

\section{The Experimental System}

The system was built in a laboratory at the Davidson Institute and included three main rails: a cycloid that was created using a circle with a radius of $16 \mathrm{~cm}$; an inclined plane with a sloping degree of $30^{\circ}$; and a pathway that was composed of a flexible rail, which could be used to change its shape (see Figures 3 and 4). A steel ball can move along each one of the rails. The steel balls are held by permanent magnets that are in a small mobile structure that can be moved along the length of the rail and change the height from which the ball can start moving. The release of the ball is performed by an electromagnet. The electromagnet creates a magnetic field that is reversed in its direction relative to the magnetic field of the permanent magnet that holds the ball. The ball is released from rest by pressing a small switch on the side of the rail. The switch is additionally connected to a PC for timing purposes. The balls on the cycloid's rail and on the inclined plane can be released simultaneously. The motion of the balls along the length of the rails can be well approximated by the sliding of a point mass, which is a conclusion we reached with the help of an experiment on the inclined plane as well as precise analysis of the motion (see above). In the next paragraph, several experiments are presented which can be performed with the system. Every experiment has a suitable method by which the ball's time in motion can be measured [2].

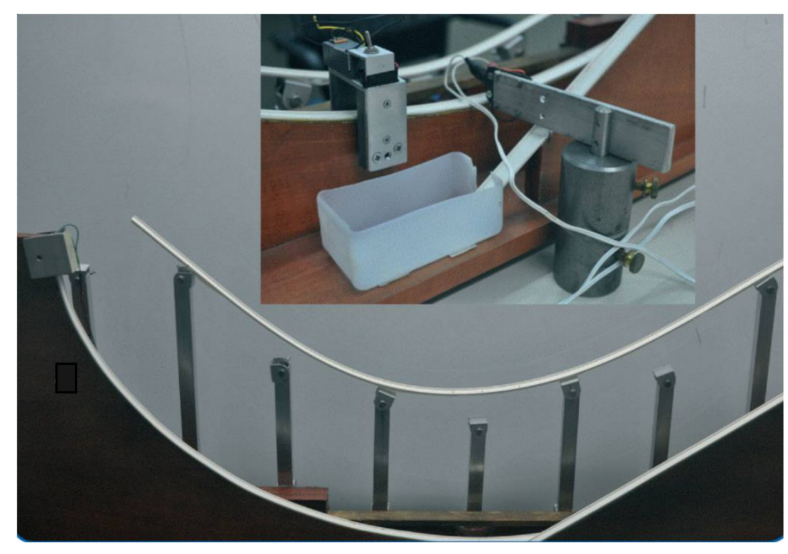

Figure 3. The experimental system: the cycloid, B; the inclined plane, $C$; and the flexible railing. In the inset, one can see the electromagnets that were used in a controlled release of the balls on railings $\mathrm{A}$ and $\mathrm{B}$. 


\section{Results and Discussion}

In this experiment, the motion time of a small metal ball whose mass is $\mathrm{m}=32.76 \mathrm{~g}$ and whose radius is $1 \mathrm{~cm}$ was measured as a function of the initial height $h$ on two different rails: the cycloid and the inclined plane (see Figure 3). In each of the rails, we changed the starting height several times and measured the total motion time from the moment of its release all the way to its bumping into the metal board that was placed at the bottom of the rail. For accuracy's sake, we averaged over three measurements for each height. (There was a sound sensor on the metal board that was at the bottom of the rail, with which the motion time was measured-see a detailed description in the frame in Figure 4). The experiment's results are presented in the graphs in Figures 2 and 5-7.

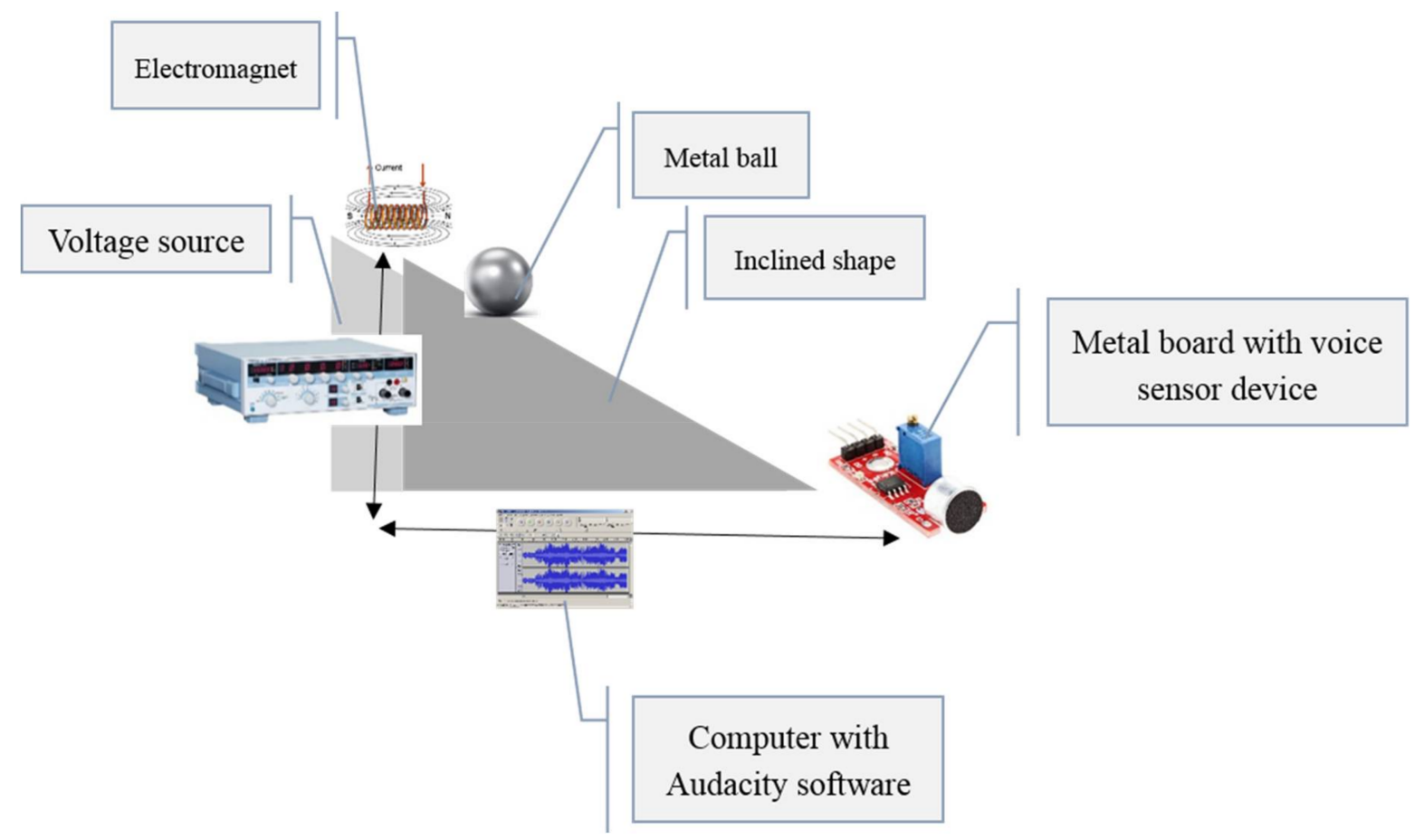

Figure 4. A schematic description of the experimental system for measuring the duration of the ball's motion on one of the rails [4].

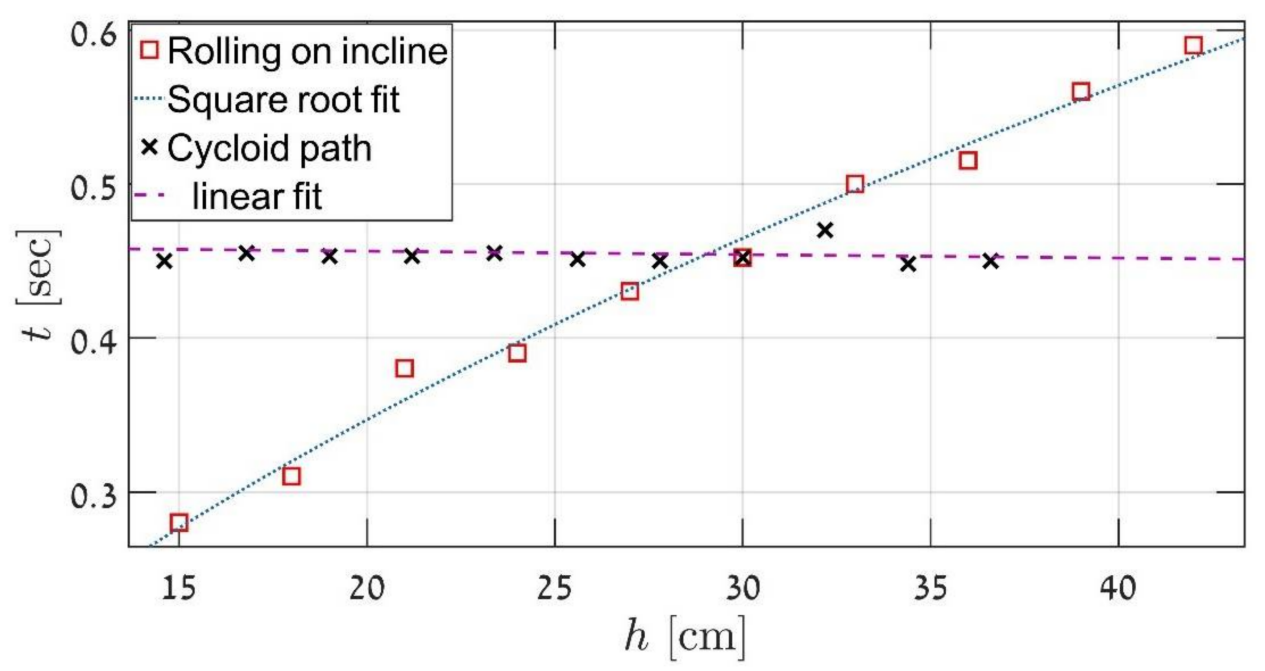

Figure 5. Duration times of the small ball's movement on the cycloid (black crosses) and on the inclined plane (red squares) as a function of the starting height as it was measured in the experiment. 
The results show that while the duration of movement in the descent of the inclined plane is dependent on the starting height, the cycloid is indeed an isochronous (of equal times) curve, meaning that the duration of the ball's motion is not dependent on the starting point along the length of the cycloid path, and it stays almost completely constant.

It is shown that with initial heights that are less than $30 \mathrm{~cm}$, the ball that moves down the descent of the inclined plane arrives first, while when $h>30 \mathrm{~cm}$, the ball that moves in the cycloid rail "wins" [1,2].

The explanation for this is that when $\mathrm{h}>30 \mathrm{~cm}$, the starting points of the two rails become closer to each other, meaning that the problem becomes identical to the brachistochrone problem (the fastest path), and the cycloid pathway is the fastest out of all the possible pathways that connect the starting point with the finishing point [3]. This could also be analyzed by progressively introducing "kinks" in an inclined path, such that in the continuum limit we get a smooth curve that is the cycloid [4,5]. A comparison between the duration time achieved in the experiment with the cycloid rail and the theoretical duration time is called for. The formula for frictionless, rotationless motion time along a cycloid is used (as is shown above):

$$
T=4 \pi \sqrt{\frac{a}{g}}
$$

where $a$ is the radius of the circle that created the cycloid. In the experiment, we measured the motion time from a certain height all the way down to the bottom of the cycloid, meaning that we measured the duration of one quarter of a motion [6-9]. The result was $0.444 \mathrm{~s}$, in comparison with the theoretical duration of one quarter of a motion $\mathrm{T} / 4=0.402 \mathrm{~s}$ (substituting $\mathrm{g}=980 \mathrm{~cm} / \mathrm{s}^{2}$ and $\mathrm{r}=16 \mathrm{~cm}$ into Equation (16)). This means that a relative deviation of $10.45 \%$ was recorded.

In our opinion, the duration time measured is bigger than the calculated time per Equation (20) mostly because the ball is not in a slipless slide regime. Part of its starting potential energy turns into circular kinetic energy while another part goes to heat due to slip friction [6-11]. This was not considered in Equation (16) or the theoretical analysis above. It is possible to compare this result with the measurement by using the optical gateway of several movements (Experiment $C$ described later on). There, the time interval recorded for a quarter of a motion was $0.451 \mathrm{~s}$. The relative deviation in this case has increased to $12.2 \%$. We estimate that this difference stems mainly from the fact that the measurement in the third experiment was performed without repetitions, as compared with the three repetitions of the measurements that were performed with the sound sensor [1-3]. When we compare the theory to the experiment in ball sliding on an incline without sliding, we can write the equation of motion of the ball sliding and the sum of momentums across the center of mass of the balls. The equations are

$$
\begin{aligned}
& \Sigma F_{x}=m g \sin \beta-f_{s}=m a \\
& \Sigma F_{y}=N-m g \cos \beta=0 \\
& \Sigma \tau_{C M}=f_{s} R=m \alpha=m \frac{a}{R}
\end{aligned}
$$

with $R$, here, denoting the ball's radius, and $a$ the acceleration. By substituting the moment of inertia of a ball around a central axis, $I=2 / 5 m R^{2}$ in Equation (2), and assuming that the ball is on the threshold of movement, the static friction force can be written as

$$
f_{s}=\mu_{s} m g \cos \beta
$$

Thus, we obtain a condition for rolling without slippage to be obtained when

$$
\mu_{s} \geq \frac{2}{7} \tan \beta
$$

Figure 7 shows the moving time of a ball as a function of the initial height. By neglecting air friction and rolling friction (i.e., a simple skid in a sloping plane), it can be shown that the final velocity of the ball starting to slide from rest is $\sqrt{2 g h}$. The use of motion formulas is accelerated and related as $x=h / \sin \beta$, and when $x$ is the distance of the slippage along the slope and $\beta$ is the slope angle, we get

$$
t_{\text {sliding }}=\frac{2 x}{v}=\frac{2 h}{\sin \beta \sqrt{2 g h}}=\frac{1}{\sin \beta} \sqrt{\frac{2 h}{g}} .
$$


Of course, we expect this theoretical time to be less than the measured time because, in reality, there is energy deposited in the rotational kinetic energy as well as energy losses due to work wasted against skid, rolling friction, and friction with the air (Figure 6).

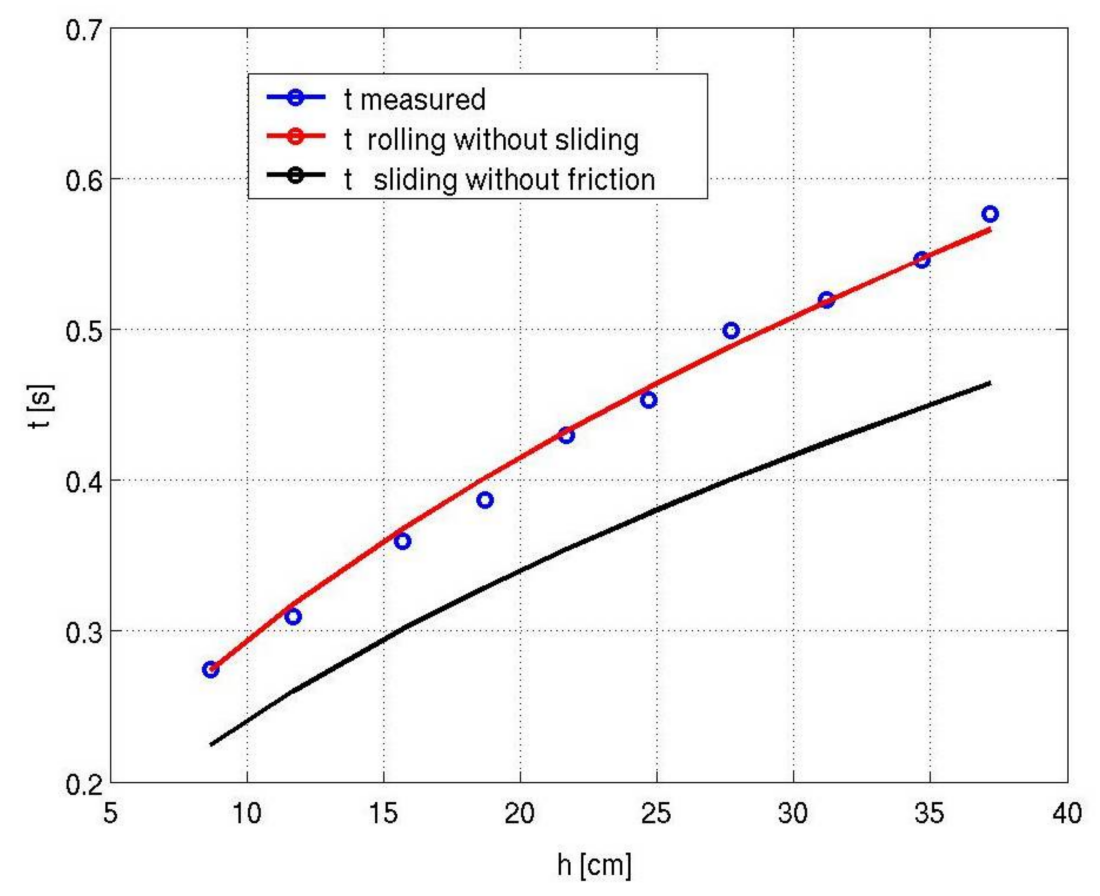

Figure 6. The motion time of the ball along the sloped plain versus the initial height. The blue dots indicate the measured time, the black line indicates the theoretical time of the plot without rolling, and the red line describes the sliding time according to Equation (19); the correspondence between the theory and the experiment is very good.

\section{The Energy Consideration}

Assuming the motion of the ball is a nonslip roll, we can calculate the real rolling time by using energy conservation and the fact that the mass center acceleration of the ball is constant. According to the law of conservation of energy, we can write

$$
m g h=\frac{m v^{2}}{2}+\frac{I \omega^{2}}{2}+\mu_{r} m g \cos \beta \cdot x
$$

in which the second expression on the right-hand side expresses the kinetic energy of the ball due to rotation around its axis and is the angular speed of the ball, and $I$ is the moment of inertia of the ball around its axis and equal to $2 / 5 m R^{2}$ when $R$ is the radius of the ball. For friction without rolling we can use the connection between the angular velocity and the linear velocity of the center of mass $v=R \omega$. The last expression on the right-hand side expresses the work that is wasted as a result of the skid friction. Rolling friction results from tiny deformations of the surface resulting from the body rolling on it [5]. The rotational friction coefficient $\mu_{r}$ is usually small compared with the coefficient of kinetic friction between the surfaces (this is the great advantage inherent in the invention of the wheel). It is worth noting that the roll is caused by the static friction between the ball and the surface, but because in the nonslip rotation the point of contact between the surface and the ball is at rest relative to the surface, there is no loss of energy as a result of the static friction [2,4,6]. However, the rolling friction still works. Of course, this also means that skid and slipless roll are mutually exclusive. Because the skid nature is sparse (meaning most of the time there is no appreciable skid), and the difference between point mass sliding and ball rotation is slight, we choose to encode the energy loss due to friction while skidding in a friction term. 
For rolling without sliding, the velocity of the center mass is $v-R \omega$ where $\omega$ is the angular velocity. Assuming the ball starts from rest and letting $\mu_{r}$ be the coefficient of friction rolling, we obtain an updated expression for the ball's motion time as a function of height $h$ :

$$
T_{\text {sliding }}=\frac{2}{\sin \alpha} \sqrt{\frac{0.7 h}{g\left(1-\mu_{r} \cot \beta\right)}} .
$$

We measured it indirectly by using the energy conservation law and the smoothness of the ball along a symmetrical inclined plane that we created using the flexible track (the slope angle of the flexible sloping plane was 34.420). The reduction in the initial potential energy should be equal to the work of the rolling friction force:

$$
\Delta E_{p}=m g \Delta h=\mu_{r} m g \cos \theta
$$

where the height difference $\Delta h$ is between the starting point of the roll and the end point [7]. Several measurements were performed and yielded $\mu_{r}=0.044$, which is a fairly reasonable value for the rolling friction coefficient.

According to energy conservation, the initial potential energy is equal to the sum of the kinetic energy, the circular kinetic energy, and the work invested in the friction of the roll (Equation (23)); therefore, we now separately express each of the energy components so that they can be calculated from $t$, which we can write as

$$
v=a t=g\left(\sin \beta-\mu_{s} \cos \beta\right) \equiv g \gamma t
$$

where $\mu_{s}$ is the static friction between the ball and rail and $\gamma=g\left(\sin \alpha-\mu_{s} \cos \alpha\right)$. Therefore, we obtain for the kinetic energy

$$
E_{k}=\frac{m v^{2}}{2}=\frac{m g^{2} \gamma^{2} t^{2}}{2} .
$$

The static coefficient of friction between the ball and the rail can be estimated from mechanical considerations (Equation (26)), and by Equation (25) we can get $\mu_{s} \geq 0.185$ for our system. Because the motion is rolling without slipping, for the purpose of calculating the energy balance, the value is selected as $\mu_{s}=0.185$. The circular kinetic energy can be expressed using angular velocity and the use of angular acceleration formulas:

$$
\omega \approx \alpha t=\frac{\mu_{s} m g \cos \beta \cdot R}{I} .
$$

Thus, in general,

$$
\omega^{2}=\frac{25}{4} \frac{\mu_{s}^{2} g^{2} \cos ^{2} \beta}{R^{2}} t^{2}
$$

We get

$$
\frac{I \omega^{2}}{2}=\frac{5}{4} m \mu_{s}^{2} g^{2} \cos ^{2} \beta t^{2}
$$

The work of rolling force friction can be expressed as follows:

$$
W_{f_{r}}=\mu_{r} m g \cos \beta \cdot x=\mu_{r} m g h \cot \beta .
$$

Using the expressions in Equations (28), (31) and (32), the overall mechanical energy can be calculated as a function of height and compared with the initial potential energy (by neglecting the air resistance) [7]. In Figure 7, it can be seen that the fit to Equation (24) is very good for all initial heights. 


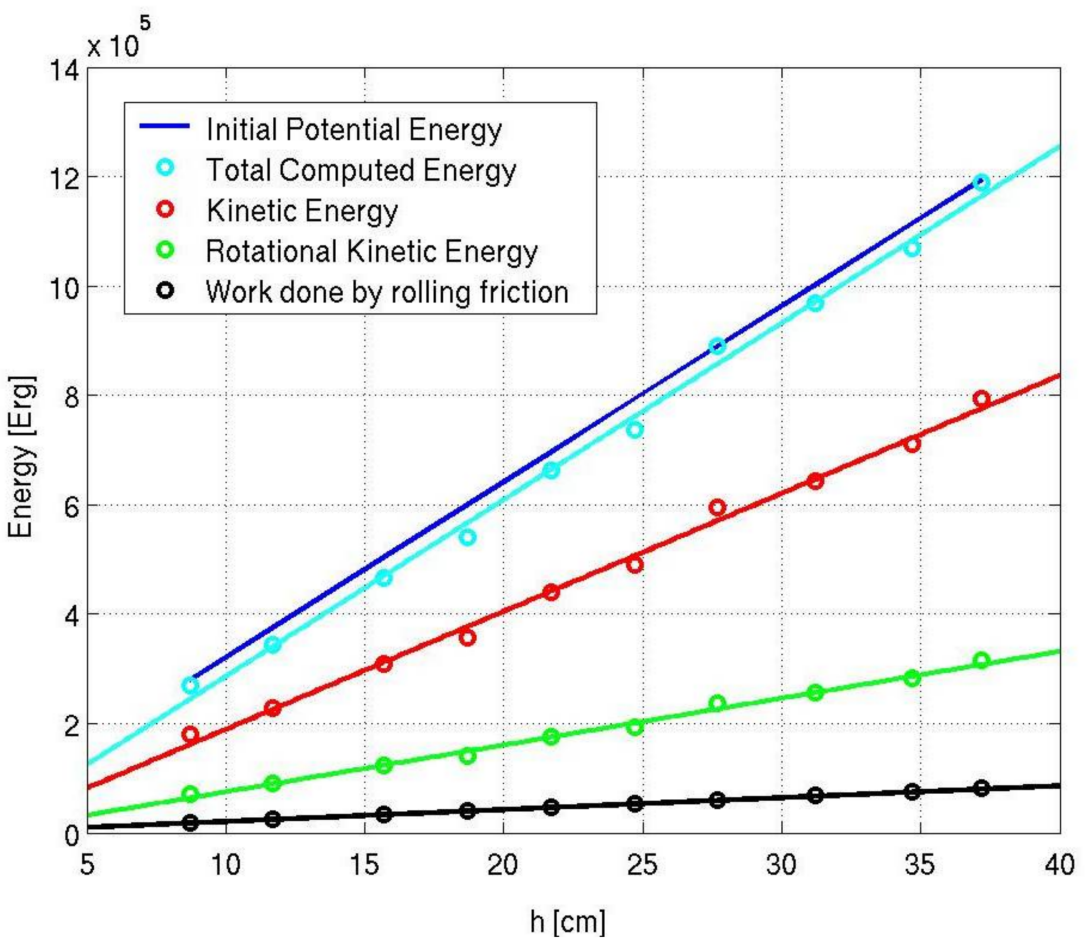

Figure 7. The total mechanical energy (in units of erg) as a function of the initial height $h$ (in $\mathrm{cm}$ ). The blue line describes the potential energy $m g h$, and the light-blue line indicates the total energy calculated using Equations (28), (31) and (32). The remaining lines indicate kinetic energy, rotational energy, and the work of rolling friction force as a function of the initial height. The correspondence between the calculation and the theory is very good and indicates that the motion of the ball down the sloping plane is a rolling without smoothing.

\section{Conclusions}

The isochronous qualities of the cycloid were studied, both analytically and experimentally. We saw that there is a discrepancy between theory and experiment, on the order of $10 \%$. We attribute this disparity to some skidding along the path, specifically in the initial stages of movement, which results in some energy loss to heat. Additional factors are the rolling deformations and the point mass sliding vs rolling ball approximation that might contribute a $1 \%$ disparity between theory and experiment. The experimental setups that were introduced are relatively inexpensive and easy to build. This might lead us to consider incorporating the cycloid into the physics curriculum, even at the high school level. The system allows investigation of the motion of small balls along curved paths and helps in demonstrating the amazing properties of a cycloid which is both brachistochrone and tautochrone. In principle, students can measure the ball velocity using a light gate and the elapsed time using Audacity software and plot the ball velocity versus time along the cycloid path. This will allow them to calculate the acceleration and will help them to understand that the final velocity depends only on the initial height and that, due to energy conservation, the acceleration and times along different ramps can be different.

Author Contributions: Yuval Ben Abu, Conceptualization, Data curation, Formal analysis, Funding acquisition, Investigation, Methodology, Project administration, Resources, Software, Supervision, Validation, Visualization, Writing — original draft, Writing - review \& editing. Ira Wolfson, Methodology, Data curation, Formal analysis, Haim Eshach, professional advice. Hezi Yizhaq, Conceptualization, Supervision and Writing-review \& editing.

Conflicts of Interest: The authors declare no conflict of interest. 


\section{Appendix A. Huygens' Proof That a Cycloid Is an Isochronic Curve 2}

The calculation of the cycle time of the cycloid plot is usually done using integral calculus and the geometric properties of the cycloid, and Huygens succeeded in solving the problem without the use of integrals but by brilliant intuition. This is a good opportunity to trace the thinking of one of the great scientists of the 20th century. First, Huygens uses a geometric feature of the cycloid that is shown in Figure A1.

The tangent $A_{t} F$ to the cycloid, which is also the direction of the velocity of the point moving on the track, cuts the circle at the highest point; $A_{t} B_{t}$ is the circle diameter, and the point $B_{t}$ is the lowest point. Therefore, the peripheral angle that rests on it is straight, and the $A_{t} F B_{t}$ triangle is a straight triangle. In this triangle, $A_{t} B_{t}=2 r \sin \alpha$, and $r$ is the radius of the circle, making $y=E_{t} B_{t}=2 r \sin ^{2} \alpha$; this produces very important characteristics of the cycloid:

$$
\sin \alpha=\sqrt{\frac{y}{2 r}}
$$

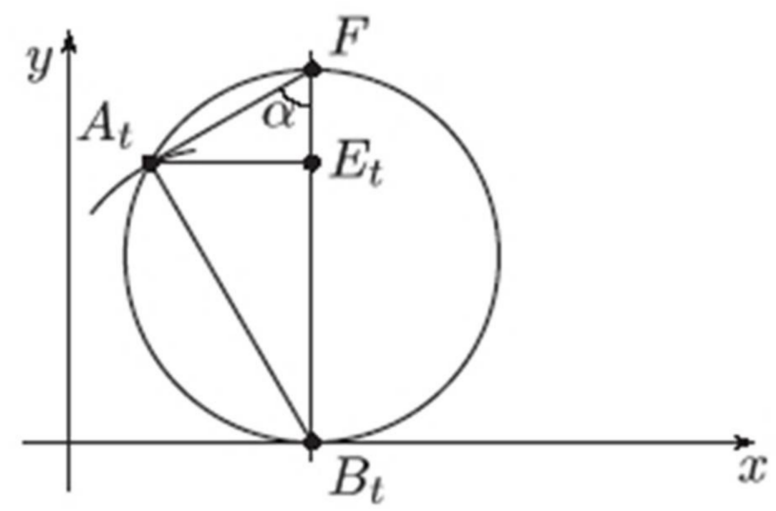

Figure A1. The tangent to a cycloid at a point $A_{t}$ passes through the point $F$ that is the highest point of the circle and creates an angle $\alpha$ with the diameter $F B_{t}$. The proof that the tangent passes at the highest point is that the direction of the tangent $A_{t} F$ is the same as the velocity of the point on the cycloid, and that velocity is equal to two equal velocities; one is the right velocity parallel to the $x$ axis and the other is the tangential velocity resulting from the circular motion. Using geometric considerations, it can be shown that $F B_{t}$ is indeed the diameter of the circle and, therefore, $A_{t} B_{t}$ is a normal for the cycloid. This feature can also be demonstrated by writing the tangent equation to the cycloid and finding its points of intersection with the circle.

Suppose that a point mass moves on the cycloid in Figures A1 and A2 and that at time $t=0$ it is at a point $C_{0}$ at distance $H$ above the plane. The goal is to find the movement time $\tau$ of the mass from the starting point $B$ to the point at the bottom of the cycloid. Assuming that there is no loss of energy and that the movement is only smooth motion, then the cycle time of the movement (up to $C_{2 \tau}$ and back) will be $T=4 \tau$. We are interested in finding out the dependence of $\tau$ on $H$. Suppose that at time $t$ the mass is at a point $C_{t}$ at distance $h$ above the plane. From the energy conservation law, we can express the speed of the mass at this point:

$$
v=\sqrt{2 g(H-h)}
$$

Now look at the projection of the position of the mass on the vertical $C_{0} B^{\prime}$. At time $t$, this projection is at point $C_{t}^{\prime}$, and at time $\tau$, it is at point $B^{\prime}$ after a vertical distance $H$. The vertical component $w$ of the velocity of the mass at point $C_{t}$ creates an angle $\alpha$ with the velocity and, therefore, $w=v \cos \alpha$. By Equation (30), $\cos \alpha=\sqrt{\frac{2 r-y}{2 r}}$, while $y=2 r-h$, and this is the $C_{t}$ from the upper right. Therefore, we obtain $\cos \alpha=\sqrt{h / 2 r}$, and for $w$,

$$
w=\sqrt{\frac{g}{r} h(H-h)}
$$

This is where Huygens' brilliance comes into play when he notices that the vertical velocity component of a circular motion is at a constant velocity in a circle with diameter $H$ similar to w (Figure A2). To prove this, we will mark the $C_{t}^{\prime \prime}$ point on the circle opposite to the point $C_{t}^{\prime}$ and the length of the segment $C_{t}^{\prime} C_{t}^{\prime \prime}$ (according 
to the Pythagorean theorem in the triangle $O C_{t}^{\prime} C_{t}^{\prime \prime}$ ). The movement of the mass on the cycloid is projected to move on the semicircle of its diameter $H$, because in both cases the vertical distance is the same, so the vertical component velocity must be the same (because the times are equal). Thus, they are similar triangles (both triangles are right-angled and they have two vertical sides, respectively). Note that the length of the vertical line coming from a point $C_{t}^{\prime}$ is $w$ and from a triangulation similarity we can draw

$$
\frac{\sqrt{h(H-h)}}{w}=\frac{H}{2 u}
$$

where $u$ is the speed along the circular motion. From Equations (32) and (33), we conclude that this speed is

$$
u=\sqrt{\frac{H^{2} g}{4 r}} .
$$

This speed is constant and equal to $\frac{H}{2} \omega$ when the angular velocity $\omega=\sqrt{\frac{g}{r}}$. In the motion of the point mass from $C_{0}$ to $B$, a half-circle is moved from point $C_{0}$ to point $B^{\prime}$ at a time equal to the half-time surrounding $\tau=2 \pi \omega$, independent of $h$. The cycle time along the cycloid will therefore be

$$
T=4 \tau=4 \pi \sqrt{\frac{r}{g}} .
$$

In conclusion, Huygens' proof is based on the idea that the velocity vector can be displayed along the cycloid as a sum of two velocities: the vertical axis is a circular motion at angular velocity independent of the starting height and the horizontal axis.

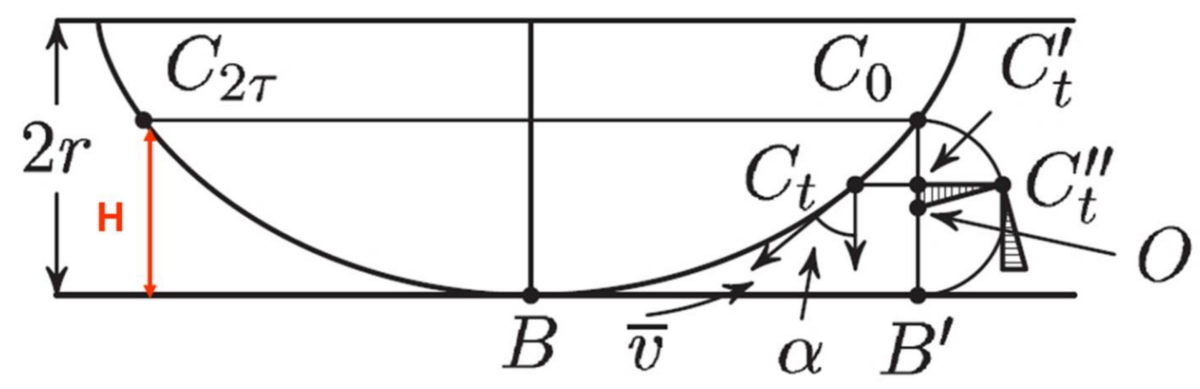

Figure A2. Huygens' proof is based on the idea that the velocity vector along the cycloid can be presented as a sum of two speeds: the vertical axis is a circular motion at angular velocity independent of the starting height along the semicircle $C_{0} C_{t}^{\prime \prime} B^{\prime}$ and the horizontal axis is a variable speed movement that increases with time.

\section{References}

1. Gindkin, S. Tale of Mathematics and Physicists, 2nd ed.; Springer: Berlin, Germany, 2007; pp. 107-193.

2. Ben-Abu, Y.; Eshach, H.; Yizhaq, H. Interweaving the Principle of Least Potential Energy in School and Introductory University Physics Courses. Symmetry 2017, 9, 45. [CrossRef]

3. Yoder, J.G. Unrolling Time; Cambridge University Press: Cambridge, UK, 1988; pp. 115-127.

4. Mungan, C.E.; Trevor, C.L. Minimum descent time along a set of connected inclined planes. Eur. J. Phys. 2017, 38, 045002. [CrossRef]

5. Theilmann, F. Approaching the Brachistochrone using inclined planes-Striving for shortest or equal travelling times. Phys. Educ. 2016, 52, 015009. [CrossRef]

6. Caravlho, S.C.; Sousa, A.S. An inexpensive technique to measure coefficient of friction with rolling solides. Phys. Teach. 2005, 43, 548-550.

7. Walker, J.; Resnick, R.; Halliday, D. Halliday \& Resnick Fundamentals of Physics, 10th ed.; John Wiley \& Sons, Inc.: Hoboken, NJ, USA, 2014.

8. Ben-Abu, Y.; Wolfson, I.; Baran, G.; Yizhaq, H. Downhill cycling symmetry breaking: How the rider foils experiment. Phys. Educ. 2017, 52, 065005. 
9. Gluck, P. Balls on an incline roll, slide and skip. Phys. Educ. 2011, 40, 206. [CrossRef]

10. Benacka, J.; Stubna, I. Ball launched against an inclined plane-An example of using recurrent sequences in school physics. Int. J. Math. Educ. Sci. Technol. 2009, 40, 696-705. [CrossRef]

11. Ben-Abu, Y. Gate Antiphase of Potassium Channel. Symmetry 2017, 9, 150. [CrossRef]

(C) 2018 by the authors. Licensee MDPI, Basel, Switzerland. This article is an open access article distributed under the terms and conditions of the Creative Commons Attribution (CC BY) license (http:/ / creativecommons.org/licenses/by/4.0/). 